\title{
Depression and Electroconvulsive Therapy: Review of Current Anesthesia Considerations
}

\author{
Peter Choi, Sergey Pisklakov ${ }^{*}$, Vasanti Tilak, Ming Xiong \\ Department of Anesthesiology and Perioperative Medicine, University of Medicine and Dentistry of \\ New Jersey, New Jersey Medical School, Newark, USA \\ Email: ${ }^{*}$ pisklase@umdnj.edu
}

Received June $19^{\text {th }}, 2013$; revised July $20^{\text {th }}, 2013$; accepted July $27^{\text {th }}, 2013$

\begin{abstract}
Copyright (C) 2013 Peter Choi et al. This is an open access article distributed under the Creative Commons Attribution License, which permits unrestricted use, distribution, and reproduction in any medium, provided the original work is properly cited.
\end{abstract}

\begin{abstract}
Electroconvulsive therapy (ECT) is becoming increasingly popular in the treatment of medication-resistant depression. Understanding the concepts behind the physiological changes brought upon by the stimulus as well as the rationale behind the various pharmacologic agents used during the procedure is crucial in providing safe and effective ECT. This review will discuss the general principles of perioperative management of anesthesia for ECT with particular focus on currently used pharmacologic agents during the procedure.
\end{abstract}

Keywords: Anesthesia for Electroconvulsive Therapy; Medications Currently Used for Anesthesia for Electroconvulsive Therapy

\section{Introduction}

ECT has been used to successfully treat a number of Axis I disorders such as major depressive disorders, mania, and schizophrenia (Kenning et al., 2012). It is a popular modality of treatment for refractory cases of many psychiatric disorders. Approximately 100,000 patients in the United States receive ECT annually. Much of this rise in popularity can be attributed to the advances in perioperative anesthesia technique during ECT, subsequently improving the safety profile of the procedure (Kellner \& Bryson 1998). This article is intended to review the current literature on perioperative anesthesia management during ECT administration.

\section{Physiological Responses to ECT}

Both the psychiatrist and the anesthesiologist must have an understanding of the physiological changes accompanying the administration of ECT to limit complications. Cardiovascular events account for most of the morbidity associated with ECT (Calarge et al., 2003). The electrical current delivered during ECT stimulates the parasympathetic nervous system via the neuronal stimulation of the vagal nerve, resulting in transient bradycardia or even asystole. This is followed by a sympathetic response due to the release of catecholamines, resulting in increases of heart rate, blood pressure, and the emergence of cardiac arrhythmias (Mayo et al., 2010). Cerebral oxygen consumption is increased due to the increased metabolic demand of the brain during the induced seizure of ECT (Uppal et al., 2010). As a result, there is a corresponding increase in blood velocity via the middle cerebral artery (Wagner et al., 2005). The cardiovascular response of increased peripheral blood pressure

${ }^{*}$ Corresponding author. may result in the auto-regulatory system being overwhelmed, which in turn may lead to increased intracranial pressures as well (Mayo et al., 2010).

Although there are no reported absolute contraindications to the use of ECT, physiological changes during the procedure warrant several relative contraindications. These may include space-occupying cerebral lesions, cerebral aneurysms, arteriovenous malformations (AVM), recent intracerebral hemorrhage, pheochromocytoma, recent myocardial infarction (Birmaher et al., 2010).

\section{Preoperative Assessment}

Preoperative assessment must begin with a thorough history and physical of the patient. The relative risk of the identified psychiatric illness left untreated must be weighed against the risk of anesthesia and ECT. Cardiovascular comorbidities should result in a thorough cardiovascular evaluation by a cardiologist, while any history of CNS pathology warrants a proper neurologic assessment (Folk et al., 2010). Preoperative fasting must be confirmed with the patient prior to general anesthetic administration. Documentation of allergies, current medications, airway examination, laboratory workup, as well as informed consent is all important components of the preoperative assessment.

\section{Concomitant Medications}

Concomitant anticonvulsant use can increase the seizure threshold or affect the clinical efficacy of ECT; therefore its dosage is generally lowered as clinically tolerated (Weiner, 2001). Benzodiazepines, commonly used as anxiolytics prior to cases, also have a propensity to increase the seizure threshold as well, and are also avoided if possible (Mayo et al., 2010; Boylan et 
al., 2010). The use of lithium during ECT has been a topic of much debate. Reports have cited associated risks of excessive cognitive disturbance, prolonged apnea, and spontaneous seizures. However a case series conducted by Dolenc and Rasmussen reports that the concomitant use of lithium with ECT may be used safely and with optimal efficacy in (Dolenk \& Rasmussen, 2005). Bupropion is known to lower the seizure threshold and thus cessation of its use is recommended as to avoid possible complications such as prolonged seizure activity and convulsive status epilepticus following ECT (Kenning 2012; Conway 2001). Theophylline use, common in patients with concurrent COPD, can also produce similar effects of prolonging seizure activity with an increased risk of post-ECT status epilepticus (Schak et al., 2001).

\section{Induction Agents}

Methohexital remains the most commonly used induction agent and is regarded as the "golden standard" by the American Psychiatry Association (Weiner, 2001). It is favored due to its rapid onset and short duration of action, as well as its low cardiac toxicity (Chanpattana, 2001). A recent systematic review showed that methohexital was superior to other anesthetics with regard to motor seizure duration (Hooten \& Rasmussen, 2008). Methohexital has the advantage of being easily titrated. However, due to a lack of availability, other induction agents have begun to become more widely used (Mayo et al., 2010).

Thiopental is also a commonly used barbiturate used as an induction agent, but has greater anticonvulsant effects thus resulting in shorter seizure durations (Mayo et al., 2010). Typical induction dose is $2-4 \mathrm{mg} / \mathrm{kg}$ (Krystal, 2010). A double blind study conducted by Hiatt showed decreased ambulatory and waking times as well as an early return of pharyngeal reflexes with methohexital as opposed to thiopental for induction (Hiatt, 1963). Induction agents such as thiopental, propofol, and ketamine have received scrutiny by various authors for their potential cardiotoxicities. However, a randomized trial of anesthetic induction agents in patients with coronary artery disease and left ventricular dysfunction suggested that these agents can be used safely as anesthetics for patients with CAD and left ventricular dysfunction. Possibly, it is the speed of injection, route, dose and experience of the clinician, rather than the property of the agent itself that influences the outcome of the anesthetic induction (Choudhury et al., 2010).

Ketamine, a derivative of phencyclidine, has a relatively high safety profile with regards to the preservation of airway reflexes and hemodynamic stability. Subsequently, these properties have led ketamine to it be the induction agent choice in certain parts of the world that have limited availability of resuscitation equipment (Morgan et al., 2012). However, ketamine is known to have a slower onset than methohexital and is notoriously known for its proclivity to induce post-emergence delirium (Spitalnic et al., 2000). Despite this well-documented complication, ketamine can be used as a means to potentiate the effects of ECT as it appears to have less anticonvulsant effects when compared to methohexital (Rasmussen et al., 1996). As a result, ketamine may be an appropriate induction agent in cases where eliciting a seizure with methohexital anesthesia is limited even with the use of the maximum available stimulus intensity (Krystal, 2010). The typical therapeutic dose ranges from 1.5 $2 \mathrm{mg} / \mathrm{kg}$ for adults (Mankad et al., 2010).

Propofol, a potent anticonvulsant, results in shorter seizure duration when compared to methohexital but still provides adequate therapeutic benefit during induction for ECT (Wagner et al., 2005). Various studies have shown that propofol is associated with a decrease in intensity and duration of seizure, despite the use of larger stimulus charges during the course of ECT. This finding did not result in any difference in treatment response with propofol during ECT compared with other induction agents such as etomidate and methohexital (Eranti et al., 2010). Due to its anticonvulsant properties, propofol may serve as a useful induction agent for adolescents and young adults who have a very low seizure threshold or prolonged seizures (25). Propofol is also associated with less nausea after ECT as well as faster recovery times (Bailine et al., 2003). Therapeutic doses range from 2 - $4 \mathrm{mg} / \mathrm{kg}$ (Walder et al., 2001).

Etomidate causes seizures of longer duration than methohexital and propofol (Mayo et al., 2010). However, its use is also associated with increased confusion and recovery time after ECT (Ding \& White, 2002). Etomidate also carries the advantage of minimally affecting hemodynamic stability, thus is well-tolerated in patients with decreased cardiac output (Jabre et al., 2009). The therapeutic dose is $0.15-0.3 \mathrm{mg} / \mathrm{kg}$ (Mayo et al., 2010).

\section{Muscle Relaxants}

Succinylcholine is the preferred muscle relaxant for ECT mainly because its fast action and short half-life make it ideal (Jabre et al., 2009). Non-depolarizing agents are used when there are contraindications to succinylcholine, such as closed angle glaucoma, a history of malignant hyperthermia, or amyotrophic lateral sclerosis (Janis et al., 1995). In most cases, succinylcholine is considered superior as a muscle relaxant agent because of the longer half-lives exhibited by non-depolarizing agents. However, a study by Hoshi et al. showed that rocuronium-sugammadex produced longer durations of seizure activity, illustrating the potential benefits of rocuronium-sugammadex as an alternative to succinylcholine (Hoshi et al., 2011).

Inhalation agents, mainly sevoflurane, have also been studied as a potential induction agent during ECT. A study by Ramsussen et al. showed that sevoflurane compared favorably with thiopental in terms of hemodynamic stability and was also associated with better postictal orientation 20 minutes after ECT. Sevoflurane also provides tocolytic activity thus making it ideal for patients who are in late-pregnancy (Calarge et al., 2003). However, some authors believe that the use of sevoflurane is time consuming to the physician with no added benefit over methohexital as an induction agent (Ding \& White, 2002).

\section{Anticholinergic Agents}

A muscarinic-anticholinergic agent is often used to counter the bradycardia and or asystole seen via vagal stimulation during ECT. This primarily occurs immediately after the initial electrical stimulation and at the end of the induced seizures (Mankad et al., 2009). Glycopyrrolate $0.2-0.4 \mathrm{mg}$, and atropine $0.4-0.8 \mathrm{mg}$ IV or $0.3-0.6 \mathrm{mg} \mathrm{IM}$, are the two most commonly used anticholinergics during ECT (Chanpattana, 2001). Glycopyrrolate is the preferred agent for most practitioners because of its inability to cross the blood-brain barrier, which can exacerbate post-ictal delirum, and its better antisialagogue properties (Eranti et al., 2009). However, atropine has also been reported to provide more protection against bradycardia and asystole when compared to glycopyrrolate (Rasmussen et al., 1999). 


\section{Cardiovascular Response Modifying Agents}

Beta blockers such as labetalol and esmolol, are two of the most commonly used agents to attenuate the ECT-induced cardiovascular response. The starting dose of labetalol is $5-10 \mathrm{mg}$ and is given usually 2 minutes prior to induction. The duration of action is about 4 - 6 hour (Chanpattana, 2001; Mankad et al., 2009). Esmolol has a faster onset, 30 - 90 seconds, with a shorter duration of action, 10 minutes, thus decreasing the likelihood of post-ictal hypotension. Although both agents possess anticonvulsant properties, esmolol is reported to shorten seizure duration more so than labetalol (Mankad et al., 2009).

Nitroglycerine may be given sublingually several minutes before ECT to attenuate hypertension especially for those patients suffering from pre-existing ischemic cardiac complications (Chanpattana, 2001; Mankad et al., 2009).

Calcium channel blockers such as nicardipine and nifedipine may also be indicated to control mean arterial pressure prior to ECT. A study showed that nifedipine did not appear to shorten the duration of the seizure during ECT and was safely used in conjunction with labetalol to control hypertension (Figiel et al., 1993). A dose-ranging study found that the optimal dose of nicardicpine was $40 \mu \mathrm{g} / \mathrm{kg}$ IV immediately before the ECT stimulus (Zhang et al., 2005).

\section{Summary}

ECT is a safe and proven treatment modality for certain Axis I psychiatric disorders refractory to pharmacologic intervention. There must be a basic understanding of the general principles of the physiologic responses that may arise as well as the pharmacologic agents used during this procedure to ensure patient safety while providing effective ECT. Communication and proper collaboration between the anesthesiologist, psychiatrist, and the patient are critical component to safe and successful ECT administration.

\section{REFERENCES}

Bruce, V., Bailine, S., Petrides, G., et al. (2003). Indications for the use of propofol in electroconvulsive therapy. Journal of ECT, 19, 129132. doi:10.1097/00124509-200309000-00002

Birmaher, B., Brent, D., et al. (1998). Summary of the practice parameters for the assessment and treatment of children and adolescents with depressive disorders. Journal of the American Academy of Child \& Adolescent Psychiatry, 37, 1234-1238. doi:10.1097/00004583-199811000-00029

Boylan, L., Haskett, R., et al. (2000). Determinants of seizure threshold in ECT: Benzodiazepine use, anesthetic dosage, and other factors. Journal of ECT, 16, 3-18. doi:10.1097/00124509-200003000-00002

Calarge, C., Crowe, R., et al. (2003). The comparative effects of sevoflurane and methohexital for electroconvulsive therapy. Journal of ECT, 19, 221. doi:10.1097/00124509-200312000-00008

Chanpattana, W. (2001). Anesthesia for ECT. German Journal of Psychiatry, 4, 33-39.

Choudhury, M., Kapoor, P., et al. (2010). A randomized trial of anesthetic induction agents in patients with coronary artery disease and left ventricular dysfunction. Annals of Cardiac Anaesthesia, 13, 217. doi:10.4103/0971-9784.69057

Conway, C., \& Nelson L. (2001). The combined use of bupropion, lithium, and venlafaxine during ECT: A case of prolonged seizure activity. Journal of ECT, 17, 216-218. doi:10.1097/00124509-200109000-00014

Ding, Z., \& White, P. (2002). Anesthesia for electroconvulsive therapy. Anesthesia \& Analgesia, 94, 1351-1364. doi:10.1097/00000539-200205000-00057

Dolenc, T., \& Rasmussen, K. (2005). The safety of electroconvulsive therapy and lithium in combination: A case series and review of the literature. Journal of ECT, 21, 165-170. doi: $10.1097 / 01 . y c t .0000174383 .96517 .77$

Eranti, S., Mogg, A., et al. (2009). Methohexitone, propofol and etomidate in electroconvulsive therapy for depression: A naturalistic comparison study. Journal of Affective Disorders, 113, 165-171. doi:10.1016/j.jad.2008.03.004

Folk, J., Kellner, C., et al. (2000). Anesthesia for electroconvulsive therapy: A review. Journal of ECT, 16, 157-170. doi:10.1097/00124509-200006000-00007

Hiatt, N. (1963). Comparative results of methohexital sodium and thiopental sodium used during out-patient general anesthesia (A doubleblind study). Journal of the American Dental Society of Anesthesiology, 10, 94.

Hooten, W., \& Rasmussen K. (2008). Effects of general anesthetic agents in adults receiving electroconvulsive therapy: A systematic review. $J$ ECT, 24, 208-223. doi:10.1097/YCT.0b013e31815bfe2a

Hoshi, H., Kadoi, Y., et al. (2011). Use of rocuronium-sugammadex, an alternative to succinylcholine, as a muscle relaxant during electroconvulsive therapy. Journal of Anesthesia, 25, 286-290. doi:10.1007/s00540-011-1095-6

Jabre, P., Combes, X., et al. (2009). Etomidate versus ketamine for rapid sequence intubation in acutely ill patients: A multicentre randomised controlled trial. The Lancet, 374, 293-300. doi:10.1016/S0140-6736(09)60949-1

Janis, K., Hess, J., et al. (1995). Substitution of mivacurium for succinylcholine for ECT in elderly patients. Canadian Journal of Anesthesia/Journal canadien d'anesthésie, 42, 612-613. doi:10.1007/BF03011879

Kenning, T., Mago, R., et al. (2012). Electroconvulsive therapy: A review of its current status. Jefferson Journal of Psychiatry, 19, 1.

Kellner, C., \& Bryson, E. (1998). Anesthesia advances add to safety of ECT. Psychiatric Times, 29, 16-18.

Mayo, C., Kaye, A., et al. (2010). Update on anesthesia considerations for electroconvulsive therapy. Middle East Journal of Anesthesiology, 20, 493-498.

Morgan, C., Curran, H., et al. (2012). Ketamine use: A review. Addiction, 107, 27-38. doi:10.1111/j.1360-0443.2011.03576.x

Rasmussen, K., Jarvis, M., et al. (1996). Ketamine anesthesia in electroconvulsive therapy. Journal of ECT, 12, 217-223.

Rasmussen, K., Jarvis, M., et al. (1999). Low-dose atropine in electroconvulsive therapy. Journal of ECT, 15, 213-221. doi:10.1097/00124509-199909000-00006

Saito, S. (2005). Anesthesia management for electroconvulsive therapy: Hemodynamic and respiratory management. Journal of Anesthesia, 19, 142-149. doi:10.1007/s00540-004-0288-7

Schak, K., Mueller, P., et al. (2008). The safety of ECT in patients with chronic obstructive pulmonary disease. Psychosomatics, 49, 208-211. doi:10.1176/appi.psy.49.3.208

Spitalnic, S., Blazes, C., et al. (2000). Conscious sedation: A primer for outpatient procedures. Hospital Physician, 36, 22-32.

Taylor, S. (2007). Electroconvulsive therapy: A review of history, patient selection, technique, and medication management. Southern Medical Journal, 100, 494-498. doi:10.1097/SMJ.0b013e318038fce0

Uppal, V., Dourish, J., et al. (2010). Anaesthesia for electroconvulsive therapy. Continuing Education in Anaesthesia, Critical Care \& Pain, 10, 192-196. doi:10.1093/bjaceaccp/mkq039

Wagner, K., Mollenberg, O., et al. (2005). Guide to anaesthetic selection for electroconvulsive therapy. CNS Drugs, 19, 745-747. doi:10.2165/00023210-200519090-00002

Walder, B., Seeck, M., et al. (2001). Propfol versus methohexital for electroconvulsive therapy: A meta-analysis. Journal of Neurosurgical Anesthesiology, 13, 93-98. doi:10.1097/00008506-200104000-00005

Figiel, G., DeLeo, B., et al. (1993). Combined use of labetalol and nifedipine in controlling the cardiovascular response from ECT. Journal of Geriatric Psychiatry and Neurology, 6, 20-24.

Zhang, Y., White, P., et al. (2005). The use of nicardipine for electroconvulsive therapy: A dose-ranging study. Anesthesia \& Analgesia, 100, 378-381. doi:10.1213/01.ANE.0000144419.44481.59 